\title{
¿CUÁN PREPARADOS LLEGAN LOS DIRECTORES ESCOLARES?: UN ANÁLISIS SOBRE SU FORMACIÓN Y TRAYECTORIAS LABORALES PREVIAS A EJERCER SU CARGO
}

\author{
Rosario Rivero ${ }^{1}$ \\ Constanza Hurtado ${ }^{2}$ \\ Ángeles Morandé ${ }^{3}$
}

\begin{abstract}
RESUMEN
La evidencia muestra que los directores escolares inciden en los aprendizajes de los alumnos. Sin embargo, en Chile se observan algunas debilidades para el ejercicio de un liderazgo efectivo. A partir de las respuestas de más de 500 directores de establecimientos subvencionados, este estudio analiza la formación y trayectorias laborales previas a ejercer el cargo por primera vez. Tanto los programas de formación, como las experiencias anteriores de liderazgo intermedio en las escuelas son espacios clave para desarrollar las habilidades y conocimientos que caracterizan la dirección eficaz. Pese a ello, uno de cada cuatro directores no cursó un posgrado especializado ni tuvo experiencia en cargos de liderazgo intermedio antes de ejercer como director por primera vez. Por su parte, solo un $13 \%$ de los casos se encontraba altamente preparado antes de comenzar a ejercer el cargo, es decir, contaba con formación y experiencia laboral especializada. Los resultados plantean el desafío de avanzar en políticas de formación de directores, en el desarrollo de una carrera directiva y en la articulación entre las políticas de liderazgo y otras de carácter educativo que fomenten una preparación - por medio de formación y experiencia laboral especializada - que favorezca el ejercicio de un liderazgo efectivo.
\end{abstract}

Palabras clave: formación directiva, liderazgo escolar, trayectoria laboral.

\section{HOW PREPARED ARE NEW SCHOOL PRINCIPALS? ANALYSIS OF TRAINING AND LABOR TRAJECTORIES PRIOR TO ASSUMING THEIR POSITION}

ABSTRACT

The evidence shows that student learning is influenced by school principals and their leadership teams. In Chile, however, school leaders' practices present some weaknesses. Based on a survey of 500 principals of subsidized schools, this study analyzes the training and work experience

1 Facultad de Educación, Universidad Diego Portales, Santiago, Chile. Contacto: rosario.rivero@udp.cl

2 Centro de Medición MIDE UC, Pontificia Universidad Católica de Chile, Santiago, Chile. Contacto: cehurtad@uc.cl

3 Investigadora independiente, Santiago, Chile. Contacto: mdmorande@uc.cl 
18 ¿CUÁN PREPARADOS LLEGAN LOS DIRECTORES ESCOLARES?: UN ANÁLISIS SOBRE SU FORMACIÓN Y TRAYECTORIAS LABORALES PREVIAS A EJERCER SU CARGO - R. Rivero, C. Hurtado, Á. Morandé

of school principals prior to their first job in this role. Graduate training programs as well as previous experience in schools with some leadership responsibilities are key opportunities to develop skills and knowledge to yield effective leadership. Nonetheless, the results show that before becoming principals, one out of four did not attend specialized graduate programs or have some type of leadership role in schools. Furthermore, only 13\% of the school principals were highly prepared for the role, with both specialized education and prior work experience. These results highlight the challenge of advancing policies that improve principals' specialized training as well as the need of articulation between educational leadership policies and other educational policies. The objective is to generate policies that will improve specialized leadership training and promote the importance of prior leadership experiences in schools, to yield effective school leadership practices.

Keywords: leadership, leadership training, work experience.

\section{Introducción}

En las últimas décadas, la evidencia internacional ha situado al liderazgo escolar como el segundo factor intraescuela más relevante para mejorar los aprendizajes de los alumnos (Barber \& Mourshed, 2007; Leithwood, Day, Sammons, Harris \& Hopkins, 2006). Al cuantificar su relevancia, este corresponde a un cuarto del efecto de las escuelas en los aprendizajes, una vez descontadas las características socioculturales de los estudiantes (Hallinger \& Heck, 1998; Marzano, Waters \& McNulty, 2005). El liderazgo escolar también se ha relacionado con logros no académicos de los alumnos, como su motivación y compromiso con el aprendizaje (Robinson, Lloyd \& Rowe, 2008; Silins \& Mulford, 2002).

Debido a su relevancia, diversas políticas buscan fortalecer y asegurar la efectividad de los líderes escolares. Entre ellas destacan la generación de estándares profesionales, el mejoramiento de los programas de formación inicial y continua, la certificación de competencias, la profesionalización de la carrera directiva y la definición de procesos de selección de los directores (Farley-Ripple, Mead, Raffel, Sherretz \& Welch, 2011; Hale \& Moorman, 2003; Pont, Nusche \& Moorman, 2009; Stevenson, 2006; Weinstein, Muñoz y Hernández, 2014). Dentro de este último grupo, varios países han avanzado en el diseño de procesos de selección, aumentando requisitos y especificando procedimientos para elegir a los directores 
escolares. Por ejemplo, Alemania, Corea, Argentina, México y Perú especifican que el título de profesor y la experiencia de docencia en el aula son requisitos mínimos para postular al cargo, mientras que en Francia, Ecuador y en algunos estados de Estados Unidos se solicitan programas especializados de formación y/o certificación de competencias directivas para ejercer como director escolar.

Coherentemente con el desarrollo de estas políticas y con las recomendaciones internacionales (Barber \& Mourshed, 2007; Pont et al., 2009), Chile ha avanzado hacia una mayor exigencia en los criterios de elegibilidad de los directores escolares, como también hacia una mayor definición de los procesos de selección para el cargo. Si bien la intención de limitar el acceso al cargo de director existe desde 1930, la normativa más reciente proviene de la Ley de Equidad y Calidad de la Educación de 2011, la cual define que los postulantes al cargo de director de establecimientos municipales deben, obligatoriamente, contar con una licenciatura de al menos ocho semestres y experiencia como docente de al menos tres años (Núñez, Weinstein y Muñoz, 2010; Weinstein y Muñoz, 2012). Adicionalmente, la ley establece que la selección de directores municipales debe realizarse a través del mecanismo de Alta Dirección Pública (ADP) para favorecer la transparencia, profesionalismo y meritocracia del proceso. Por el contrario, el proceso de selección de directores para establecimientos particulares subvencionados y particulares pagados no está regulado.

Por su parte, desde la literatura, diversas investigaciones han estudiado las características de los directores chilenos, evidenciando que estos tienen una edad avanzada, vasta experiencia en el cargo y alto nivel de formación continua, si se los compara con otros países Latinoamericanos ${ }^{4}$. A su vez, investigaciones recientes han estudiado distintas dimensiones de los procesos de selección de directores vía ADP, analizando la valoración del proceso según sus participantes, las características de directores seleccionados y el potencial efecto de los directores elegidos bajo este mecanismo en el aprendizaje de los alumnos

Específicamente, los datos muestran que, en promedio, los directores chilenos tienen 52 años, cuentan con 13 años de experiencia en el cargo y un 48\% cursó algún programa de magíster (Weinstein y Muñoz, 2012). 
(Agencia de la Calidad, 2016; Centro de Desarrollo de Liderazgo Educativo, CEDLE, 2016; Errázuriz, Kutscher y Williamson 2017).

Sin embargo, más allá de estos aportes, es escasa la investigación nacional que estudie y caracterice la formación y las trayectorias laborales de los directores antes de que ejercieran este cargo por primera vez, desconociendo cuán preparados llegan para ejercer un liderazgo efectivo en los establecimientos subvencionados del país. Considerando la escasa presencia de procesos de inducción de directores y la baja exigencia de requisitos para postular al cargo en el caso chileno, es de esperar que la formación y las trayectorias laborales previas sean los principales espacios de generación de habilidades y competencias necesarias para ser un líder efectivo ${ }^{5}$.

De esta forma, considerando la relevancia del liderazgo escolar, la incipiente investigación en el área, la baja exigencia de los requisitos para ser director y las diferencias de normativa de los procesos de selección de directores escolares entre el sector municipal y el particular subvencionado, el presente estudio analiza cuán preparados se encuentran los directores de establecimientos subvencionados para ejercer el rol de forma satisfactoria, al momento de ser seleccionados por primera vez para el cargo.

Específicamente, mediante el análisis de una encuesta nacional realizada a directores en ejercicio en 2015 y en coherencia con la pregunta de investigación, los objetivos específicos de este estudio son:

- analizar el grado preparación de los directores antes de ejercer el cargo, según su formación, trayectoria laboral en establecimientos y tipos y años de experiencia laboral;

- analizar si existen diferencias en el grado de preparación de directores en el tiempo y;

- analizar si existen diferencias en el grado de la preparación de directores en el sector municipal, luego de la implementación de la política de selección de directores vía ADP.

5 En el caso chileno el Marco para la buena dirección y el liderazgo escolar (MBDLE) es la normativa que orienta las habilidades, conocimientos y prácticas que deben realizar los lideres escolares efectivos. 
Conocer cuán preparados están los directores para ejercer su cargo de forma satisfactoria es crucial para mejorar su formación y liderazgo, así como también para el diseño e implementación de políticas que buscan fortalecerlo, ya sea a través del desarrollo de estándares, mejoras a los procesos de selección, creación de planes de desarrollo o carrera directiva, y para la articulación de todas las políticas en el área.

La siguiente sección presenta los componentes del Marco para la buena dirección y el liderazgo escolar (MBDLE), instrumento que orienta el liderazgo escolar efectivo. Luego, en la tercera sección se presenta una revisión acerca de qué sabemos de la formación, experiencia y trayectoria laboral de los directores en establecimientos. En la cuarta, se describe la normativa vigente sobre políticas de selección de directores de establecimientos subvencionados. La sección cinco presenta antecedentes acerca de la recolección de datos, características del instrumento y los aspectos metodológicos del estudio; mientras que en la sexta se describen los resultados. La sección siete se dedica a la conclusión y discusión.

\section{Marco de referencia}

\subsection{Marco de la buena dirección de liderazgo escolar (MBDLE)}

Lo que se entiende en Chile por un liderazgo escolar efectivo fue recogido en el Marco de la buena dirección (MBD) propuesto por el Ministerio de Educación en el año 2005. Este fue actualizado en 2015 por el Marco de la buena dirección de liderazgo escolar (MBDLE). Este instrumento no es prescriptivo para los equipos directivos de los establecimientos. Su objetivo principal más bien es orientar el desarrollo de un liderazgo escolar efectivo en contextos diversos, así como también los procesos de formación especializada, de modo de fortalecer a los equipos directivos del país. Su base se encuentra en avances y experiencias en materia de gestión y liderazgo escolar (Ministerio de Educación, Mineduc, 2015).

Este marco define las áreas en que los equipos directivos deberían actuar (prácticas), conocimientos que deberían tener y 
habilidades y principios deseables para su labor. Si bien el MBDLE no es un instrumento prescriptivo, como ya se mencionó, explicita prácticas concretas deseables, independientes de la función directiva específica (Day et al., 2009; Marfán, Muñoz y Weinstein, 2012), para liderar y gestionar una institución escolar de manera efectiva, siendo una de ellas la de director del establecimiento. El documento se inclina por una mayor centralidad de las prácticas, por definición contextualizadas, en contraste con el concepto de competencia. Así el énfasis de las prácticas sugeridas varía según las características de los establecimientos como su vulnerabilidad, tipo o la etapa de mejoramiento en que se encuentra (Day et al., 2009; Marfán et.al. 2012). Las prácticas describen lo que es necesario saber hacer, en términos amplios, para liderar y gestionar una institución escolar de manera efectiva. Estas se agrupan en cinco dimensiones:

- construir e implementar una visión estratégica compartida;

- desarrollar capacidades profesionales;

- liderar procesos de enseñanza y aprendizaje;

- gestionar la convivencia y la participación de la comunidad escolar;

- desarrollar y gestionar el establecimiento escolar.

A su vez, el documento señala que para dar cumplimiento a estas prácticas se requieren recursos personales organizados en tres ámbitos:

- principios, referidos al actuar el ético, trasmitir y generar confianza a través de su comportamiento, la justicia social y la integridad;

- habilidades, que incluyen una visión estratégica, trabajo en equipo, comunicación efectiva, capacidad de negociación, aprendizaje permanente, flexibilidad, empatía, sentido de autoeficacia y resiliencia y;

- conocimientos profesionales, vinculados con el liderazgo escolar, inclusión y equidad, mejoramiento y cambio escolar, currículo, evaluación, prácticas de enseñanza-aprendizaje, desarrollo profesional, políticas nacionales de educación, normativa nacional y local, y gestión de proyectos (Mineduc, 2015). 
Por lo tanto, para ejercer el cargo de forma satisfactoria, los directores deben estar preparados en múltiples tareas y funciones.

En la actualidad el MBDLE puede ser usado para la evaluación de los programas de formación, conocer el grado de preparación de los directores para ejercer un liderazgo efectivo; así como también para el desarrollo de programas de formación especializada en gestión y liderazgo escolar y el desarrollo de políticas para su fortalecimiento. Sin embargo, hay escasa evidencia acerca de los usos del marco en estos contextos. Desde las políticas educativas, la reciente Ley de Carrera Docente establece que solo pueden ser directores los profesores que tengan una evaluación sobresaliente en habilidades, conocimientos y prácticas definidas en el MBDLE (tramo avanzado de la carrera).

\section{2. ¿Qué sabemos sobre la preparación de los directores} antes de ejercer su cargo por primera vez?

La investigación internacional evidencia que la formación y las trayectorias laborales de los directores se vinculan con su efectividad. Sin embargo, para el caso de la formación la evidencia no es concluyente, ya que algunos estudios internacionales encuentran que la formación especializada de los directores se asocia positivamente con el aprendizaje de los estudiantes (Clark, Martorell \& Rockoff, 2009; Loeb, Kalogrides \& Horng, 2010), mientras que otros estudios concluyen que programas especializados no afectan el desempeño de los directores (Hale \& Moorman, 2003). Esta revisión permite concluir que no hay consenso acerca de la relación entre la formación especializada de los directores y su efectividad.

Por otra parte, al indagar con mayor profundidad en los estudios que encuentran una asociación positiva entre formación y aprendizaje de los alumnos, se evidencia que solo aquellos programas de formación con un fuerte foco en las experiencias prácticas en las escuelas se asocian con el ejercicio de un liderazgo efectivo (Militello, Gajda \& Bowers, 2009).

Respecto de las trayectorias laborales de los directores en los establecimientos, los estudios internacionales coinciden en que 
una mayor experiencia en cargos directivos es positiva para ejercer un liderazgo efectivo (Bowers \& Bradford, 2014; Rice, 2010). Coherentemente, sostenedores y empleadores prefieren seleccionar directores que se hayan desempeñado en cargos de liderazgo en establecimientos anteriormente (Gates, Ringel \& Santibáñez, 2003). Por otra parte, la relación entre la experiencia como docente en aula y el aprendizaje de los alumnos es menos clara. Así, mientras algunos autores evidencian una relación positiva entre la experiencia como docente en aula en la misma escuela y el aprendizaje (Bowers \& Bradford, 2014), otros señalan que la experiencia en el cargo docente no incidiría en el desempeño como director (Rice, 2010).

Por su parte, la investigación nacional provee alguna evidencia acerca de la formación y experiencia en otros cargos ejercidos por los directores. Así, cursar programas de formación continua, de al menos un año de duración, es altamente probable entre directores escolares. Específicamente, el 70\% completó algún postítulo y el $48 \%$ un magíster, siendo ambos más frecuentes en directores de establecimientos municipales ${ }^{6}$. Estas diferencias, según la dependencia del establecimiento, se explican, en parte, porque la normativa de los municipales privilegia la selección de postulantes que completaron estos programas. A su vez, la mayoría de los directores municipales (83\%) se especializó en áreas de liderazgo y gestión antes de comenzar a ejercer (CEDLE, 2016). Si bien, para el caso chileno, no existe evidencia acerca de la asociación entre la formación y la efectividad de los directores en relación con el aprendizaje de los alumnos, un $50 \%$ de los directores considera que la formación continua en áreas de liderazgo y gestión contribuye a un desempeño satisfactorio de su rol (CEDLE, 2016).

En términos de las trayectorias laborales, la investigación nacional evidencia que el rol directivo suele ser la etapa final de la carrera docente (Weinstein et al., 2014). En cuanto a los cargos

6 En el caso de postítulos, un $89 \%$ de los directores de establecimientos municipales los han cursado y $57 \%$ de los colegios particulares subvencionados. Los porcentajes corresponden a $65 \%$ y $35 \%$, respectivamente, para el estudio de magíster. Estos registros no son excluyentes entre sí, ya que un mismo director puede tener ambos tipos de formación (Muñoz y Marfán, 2012). 
ejercidos anteriormente al de director, Weinstein, Cuéllar, Hernández y Fernández (2016) estudiaron los procesos de socialización de 76 directores novatos de establecimientos municipales elegidos vía ADP y concluyeron que todos ejercieron como docentes de aula y que un $80 \%$ ocupó cargos de liderazgo intermedio anteriormente. De acuerdo con la percepción de estos directores, su experiencia en cargos directivos, específicamente la de jefe de UTP, es la más significativa para la preparación al cargo de director. También lo son el carácter insider/outsider ${ }^{7}$ al cargo, la dependencia de origen y los programas de formación que han cursado. Otra evidencia disponible es la encuesta Voz de los directores 2016 realizada a 600 directores escolares de todas las dependencias administrativas (CEDLE, 2016). Los resultados muestran que los estos perciben que la docencia es la experiencia más significativa para ejercer el rol, seguida de ejercer cargos de liderazgo intermedio.

En cuanto a los directores del sector particular subvencionado, a partir de una encuesta a 649 directores, representativa a nivel nacional, Weinstein y Muñoz (2012) concluyeron que estos experimentan una menor progresión de cargos previos en establecimientos al compararlos con los de establecimientos municipales, siendo más común que en el sector privado pasen directamente de la docencia a la dirección (20\% en municipales versus $43 \%$ en los particulares). A su vez, los directores municipales contarían con más años de experiencia previa, llegando al cargo a edades más avanzadas. Según los autores, estas diferencias se explicarían por menores requisitos y competitividad en el sector subvencionado, como también por la mayor informalidad de los procesos de selección en este grupo.

Por último, algunos estudios evalúan la implementación del sistema de selección por ADP, exclusiva del sector municipal. Estos detallan las características de los directores seleccionados y el impacto del elegido sobre los aprendizajes de los alumnos. En relación con los cambios de las características de los directores, los estudios muestran

El carácter insider al cargo se refiere a cuando el director seleccionado trabajaba previamente en el establecimiento. Por el contrario, el carácter outsider se refiere a cuando el director seleccionado trabajaba previamente en un establecimiento distinto al cual fue seleccionado como director. 
26 ¿CUÁN PREPARADOS LLEGAN LOS DIRECTORES ESCOLARES?: UN ANÁLISIS SOBRE SU FORMACIÓN Y TRAYECTORIAS LABORALES PREVIAS A EJERCER SU CARGO - R. Rivero, C. Hurtado, Á. Morandé

que, en promedio, los directores seleccionados son dos años más jóvenes, tienen tres años menos de experiencia en establecimientos y cinco menos en el establecimiento para el que fueron seleccionados como director, en comparación con aquellos de establecimientos municipales no seleccionados por ADP (Agencia de la Calidad de la Educación, 2016). Cuando la comparación es respecto del director anterior del mismo establecimiento, se observa que los directores seleccionados vía ADP son, en promedio, siete años más jóvenes, tienen cuatro años menos de experiencia laboral y seis años menos en el establecimiento para el cual fueron seleccionados como director. Los datos también muestran que el 34\% de los directores escogidos por ADP completó programas de pedagogía media versus el 19\% de los directores no seleccionados por esta vía. Finalmente, en relación con la asociación entre el sistema de selección de los directores y los resultados de aprendizajes de los alumnos, no se encuentran diferencias en los resultados de aprendizaje de los alumnos en $4^{\circ}$ año básico y $8^{\circ}$ año básico en Simce, ni en el desempeño de los alumnos en la Prueba de Selección Universitaria, PSU (Agencia de la Calidad de la Educación, 2016).

\subsection{Políticas nacionales que definen los procesos de selección de directores}

Tras el proceso de municipalización en la década de los ochenta comenzó la descentralización de la selección de directores de establecimientos públicos. Se atribuye a esta atomización, una pérdida de prestigio y centralidad del rol y la instauración del cargo como el fin de la trayectoria docente (Núñez et al., 2010). Tras este periodo, la selección de los directores se normó en el Estatuto Docente de 1991, la que definió como requisitos deseables para el cargo de director, el título docente y la formación adicional en administración, supervisión o evaluación. Este estatuto promovió procesos de selección basados en la demostración de habilidades de los postulantes, incentivando la selección por mérito de directores municipales y limitando la discrecionalidad de los municipios. El año 1995 se fijó una duración máxima de cinco años para el cargo. Posteriormente, en 2005 estas medidas (requisitos deseables y duración máxima) se indicaron como obligatorias para la educación municipal, fijándose un calendario 
gradual de reemplazo del universo de directivos. En el año 2009, la Ley General de Educación (LGE) insistió en la obligatoriedad de selección vía concurso en el sector público (CEDLE, 2017; Núñez et al., 2010).

Luego, en el año 2011, la Ley de Equidad y Calidad de la Educación profesionalizó aún más el sistema, instalando un procedimiento público y transparente, donde cada etapa de selección de directores es abierta desde el municipio, lo que genera un perfil idóneo al cargo. Para llevarlo a cabo, se conforma una comisión calificadora independiente, integrada por un consejero de ADP, el jefe de educación y un docente destacado del municipio. Tras evaluar a los candidatos, la comisión presenta una nómina de tres a cinco candidatos al sostenedor, quien finalmente elige al director. Por su parte, el Ministerio de Educación (Mineduc) provee un banco de perfiles para orientar a municipios según sus características, considerando la ruralidad, nivel de enseñanza y desempeño del establecimiento. El perfil general establece que los postulantes deben, obligatoriamente, contar con el título de docencia y tener al menos tres años de experiencia como docentes de aula ${ }^{8}$, perfeccionamiento en áreas afines a la dirección escolar y al menos cinco años de experiencia. Se mencionan como características deseables la experiencia en cargos de dirección, coordinación y/o jefatura, y la demostración de competencias, como liderazgo pedagógico, gestión de personas y de logro.

En relación con la implementación, la ley dictó que al año 2016 todos los directores de establecimientos municipales debían ser seleccionados por esta vía. Sin embargo, el avance de la política al 2016 era de 65\%, concentrándose la selección de directores por ADP en establecimientos de áreas urbanas, de menor vulnerabilidad y mayor tamaño (Agencia de la Calidad de la Educación, 2016; Errázuriz et al., 2016). Esto se atribuye a una excesiva dependencia de las capacidades de los municipios para la implementación de la política (Grupo Educativo, 2015). En contraste, sobresale positivamente que

Se eximen de este requisito quienes cuentan con una licenciatura y hayan ejercido al menos tres años en un establecimiento educacional. 
alrededor de un $60 \%$ de los procesos da como resultado la elección de directores que no ejercían el cargo en el mismo establecimiento, un alto número de postulaciones (20 en promedio en zonas urbanas) y una alta valoración de los concursos por parte de los participantes (CEDLE, 2017; Weinstein et al., 2014).

Por último, la reciente Ley $N^{\circ} 20.903$ de Carrera Docente establece que, desde el año 2017, solo quienes se encuentren en

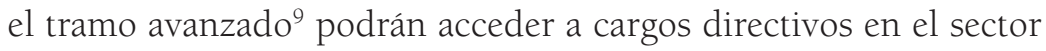
municipal. Este tramo se caracteriza por la consecución por parte de los docentes de las habilidades señaladas en el Marco de la buena enseñanza, como habilidades para la enseñanza del aula, reflexión pedagógica y participación en roles de mentoría y diseño de programas de mejoramiento escolar.

En relación con el proceso de selección de directores de establecimientos particulares subvencionados y particulares pagados la normativa no regula su funcionamiento quedando a discreción de los sostenedores la elección del director.

\section{Aspectos metodológicos, muestra y variables}

\subsection{Recolección de datos}

Este artículo se basa en un estudio de carácter cuantitativo. Se reportan resultados descriptivos sobre la base de la aplicación de la encuesta de cobertura nacional La voz de los directores, que se aplica anualmente desde el año 2013, y cuya población objetivo son todos los directores y directoras del país. Esta encuesta es un instrumento que consta de dos secciones. La primera contiene preguntas cerradas que abordan la percepción de los directores acerca de la calidad de la educación en general y sobre algunas políticas educativas recientes; la segunda sección varía año a año, es decir, va cambiando en términos del número de preguntas, así como también en cuanto a las temáticas que se abordan. La versión utilizada en este estudio (2015) contuvo varios ítems relacionados con la formación y la experiencia de los

Docentes con cuatro años de experiencia que obtienen el logro más alto en el instrumento portafolio (A). 
directores, permitiendo distinguir la trayectoria laboral antes de ejercer el cargo por primera vez, aun cuando estos hayan trabajado como directores en distintos establecimientos. Este instrumento ha sido debidamente testeado todos los años entre directivos en términos de su comprensión, tiempo de aplicación y validez. La aplicación de la encuesta se realizó entre noviembre de 2015, y enero del 2016, mediante un formato autoaplicado y en línea, y ha estado a cargo de instituciones especializadas. El cuestionario es enviado por correo electrónico al universo de directores, de enseñanza básica y media, que poseen una dirección válida. Luego se reenvía electrónicamente buscando ampliar la tasa de respuesta lograda inicialmente. Finalmente, se procede a llamar telefónicamente a los establecimientos insistiendo en la invitación al director a completar la encuesta y enviarla. Este paso final se realiza mediante un muestreo aleatorio por cuotas, priorizando a determinados grupos de establecimientos para lograr una muestra proporcional a la distribución del universo de establecimientos según su dependencia administrativa.

Entre los datos disponibles de la encuesta anual Voz de los directores del año 2015, hay 504 casos de directores de establecimientos municipales y particulares subvencionados, es decir, el 5,7\% de los directores de establecimientos subvencionados en Chile, de los cuales más del 90\% han sido directores en más de uno. Se les preguntó acerca de su formación, años de experiencia laboral, cargos ocupados en establecimientos y tiempo ejerciendo cada rol, antes de llegar al cargo por primera vez. El error muestral puede estimarse, bajo el supuesto de que no hay diferencias significativas entre las características de la muestra y del universo, en 4\% con un 95\% de confianza.

\subsection{Dimensiones de análisis}

Posteriormente, se procedió a realizar un análisis estadístico descriptivo de los datos. En primer lugar, con el objetivo de construir una variable que midiera el grado preparación de los directores para ejercer su cargo por primera vez, se analizó su formación continua y sus trayectorias laborales en establecimientos antes de llegar al cargo. Primero, se estudió la variable "Formación continua", distinguiendo 
30 ¿CUÁN PREPARADOS LLEGAN LOS DIRECTORES ESCOLARES?: UN ANÁLISIS SOBRE SU FORMACIÓN Y TRAYECTORIAS LABORALES PREVIAS A EJERCER SU CARGO - R. Rivero, C. Hurtado, Á. Morandé

el grado más alto alcanzado (curso; diplomado o postítulo; magíster o doctorado), ya sea en liderazgo o gestión, o en otra área de educación ${ }^{10}$. En segundo lugar, se estudiaron las trayectorias laborales en establecimientos, según el tipo de cargo ejercido antes de ser director. Se construyó la variable "Trayectoria" que distingue tres grupos:

- directores que no trabajaron como profesores de aula ni como líderes intermedios en escuelas (por ejemplo, jefatura de UTP y/o subdirección), llamado "No profesor, no liderazgo intermedio";

- directores que no trabajaron en cargos de liderazgo intermedio y sí como docentes de aula, llamado "No liderazgo intermedio" y;

- directores que ejercieron como profesores y como líderes intermedios, llamado "Profesor y liderazgo intermedio".

A partir de estas dos variables ("Formación continua" y "Trayectoria") se elaboró una nueva variable llamada "Nivel de preparación", la cual clasifica a todos los directores según la formación continua cursada y los cargos ejercidos en establecimientos antes de ser directores por primera vez. El Cuadro 1 ilustra la clasificación de los directores según los criterios para definir su nivel de preparación. Se clasificaron como directores con nivel "alto de preparación" a quienes ejercieron previamente como docentes de aula y en cargos de liderazgo intermedio y que adicionalmente cursaron un magíster o doctorado especializado en gestión y/o liderazgo. Por otra parte, se catalogaron como de "mediana preparación" a dos grupos de directores: aquellos que ejercieron cargos de liderazgo intermedio y no cuentan con un posgrado en liderazgo; y aquellos que no ejercieron cargos de liderazgo intermedio, pero sí completaron un posgrado especializado. Por último, se clasificaron como de "baja preparación" a directores que no se desempeñaron en cargos de liderazgo intermedio ni cursaron un posgrado en gestión y/o liderazgo.

10 Se desconoce si es que los programas de formación continua se completaron antes o después de comenzar a ejercer el cargo, sin embargo, se asume que se cursaron antes de empezar a ser directores. Esto se apoya en la evidencia nacional que señala que los programas chilenos se concentran en profesores que aspiran a ser directores (Muñoz y Marfán, 2012). 
Cuadro 1

Nivel de preparación de los directores previa a ejercer el cargo

\begin{tabular}{llll}
\hline $\begin{array}{l}\text { Formación continua / } \\
\text { Trayectoria laboral en } \\
\text { stablecimientos }\end{array}$ & $\begin{array}{l}\text { No docente de aula, } \\
\text { no liderazgo } \\
\text { intermedio }\end{array}$ & $\begin{array}{l}\text { Docente de aula, } \\
\text { no liderazgo } \\
\text { intermedio }\end{array}$ & $\begin{array}{l}\text { Docente de } \\
\text { aula y liderazgo } \\
\text { intermedio }\end{array}$ \\
\hline $\begin{array}{l}\text { Sin formación continua. } \\
\text { Diplomado, curso de } \\
\text { perfeccionamiento o } \\
\text { postítulo en liderazgo o } \\
\text { educación. }\end{array}$ & Baja preparación \\
\hline $\begin{array}{l}\text { Magíster o doctorado en } \\
\text { educación. }\end{array}$ & $\begin{array}{c}\text { Mediana } \\
\text { preparación }\end{array}$ \\
\hline $\begin{array}{l}\text { Magíster o doctorado en } \\
\text { liderazgo o gestión }\end{array}$ & Mediana preparación & \\
\hline
\end{tabular}

Fuente: Elaboración propia.

A su vez, para caracterizar a estos grupos de directores organizados según su grado de preparación para ejercer el cargo antes de ser director, se estudiaron los tipos y años de experiencia laboral previa en establecimientos. La variable "Tipos de experiencia" clasifica en:

- fuera del ámbito educacional;

- en el ámbito educacional (pero no en establecimientos educacionales) y;

- en establecimientos y las edades asociadas a cada etapa.

La variable "Tiempo en establecimientos" distingue tres categorías, según los años de experiencia antes del cargo:

- directores que trabajaron 10 o menos años en establecimientos, llamada "fast";

- directores que trabajaron entre 11 a 24 años en establecimientos, llamada "medium" y;

- directores que trabajaron 25 o más años, llamada "low".

Luego, para analizar la preparación para ejercer el cargo en la última década, se elaboraron dos variables en función del año en 
32 ¿CUÁN PREPARADOS LLEGAN LOS DIRECTORES ESCOLARES?: UN ANÁLISIS SOBRE SU FORMACIÓN Y TRAYECTORIAS LABORALES PREVIAS A EJERCER SU CARGO - R. Rivero, C. Hurtado, Á. Morandé

que los directores fueron nombrados en el cargo por primera vez. La variable "Año de nombramiento", distingue a su vez dos categorías: antes del año 2005; y 2005 o después.

Considerando que la implementación de ADP comenzó a regir el año $2012^{11}$, se construyó una variable llamada "Selección ADP", agrupando en "seleccionado" a aquellos directores del sector municipal que fueron seleccionados desde el año 2012 en adelante y en "no seleccionados" a aquellos elegidos antes de $2012^{12}$.

\subsection{Marco muestral y características de la muestra}

El universo del estudio corresponde a 8.864 directores de establecimientos subvencionados (Mineduc, 2015). La Tabla 1 presenta descriptivos de las características de la muestra y su comparación con los datos disponibles del universo de directores (Mineduc, 2015). El promedio de edad de los encuestados es de 56 años y es levemente superior a los 52 años promedio a nivel nacional, sin embargo, hay menor dispersión de la edad en la muestra que en la población (8 y 11 años de desviación estándar, respectivamente). La proporción de hombres es superior en la muestra que en el universo ( $52 \%$ versus $38 \%$ ). Respecto del promedio de años de experiencia en establecimientos, el promedio de la muestra es superior a la población (30 versus 24 años promedio). En cuanto a los establecimientos, la proporción de establecimientos municipales es equivalente entre la muestra y la distribución nacional ${ }^{13}$. Por último, la distribución por nivel de enseñanza presenta una mayor concentración de establecimientos de enseñanza básica en la muestra si se le compara con la distribución nacional.

11 Si bien la selección por ADP no rige para los establecimientos particulares subvencionados, es posible que su incorporación repercuta en la selección de directores de esta dependencia (modificando el perfil de quienes postulan al cargo, o patrones de rotación entre establecimientos), sin embargo, el porcentaje de casos de esta dependencia nombrado desde el año 2012 es bajo (8\%), impidiendo generar comparaciones válidas entre grupos.

12 Debido a que una fracción de directores municipales elegidos vía ADP, ejercían como directores en el mismo establecimiento ( $40 \%$, según estudio de la Agencia de la Calidad de la Educación) es posible que una parte de los directores, haya sido elegido por este mismo mecanismo.

13 Esta cifra corresponde a la proporción de establecimientos que reciben subvención del Estado y que imparten enseñanza básica o media regular. Ministerio de Educación Chile, 2015. 
Es importante considerar, por lo tanto, que una de las limitaciones de este estudio es que la muestra de directores subvencionados es levemente mayor y cuenta con más años de experiencia laboral que el universo, como también que tiene una mayor concentración de hombres y de directores de establecimientos de básica, por lo que la interpretación de los resultados debe ser realizada con cautela. Sin embargo, este estudio es único en términos de la recolección de datos, ya que cuenta con información de más de 500 directores de establecimientos subvencionados y son leves las diferencias entre la muestra y el universo.

Tabla 1

Características del universo y muestra

\begin{tabular}{|c|c|c|c|c|c|c|}
\hline \multirow[b]{2}{*}{ Variable } & \multicolumn{3}{|c|}{ Universo } & \multicolumn{3}{|c|}{ Muestra } \\
\hline & $\mathrm{N} \%$ promedio & D.E. & $\%$ & No/ promedio & D.E. & $\%$ \\
\hline \multicolumn{7}{|l|}{ Género } \\
\hline Hombre & 3.386 & & $38 \%$ & 260 & & $52 \%$ \\
\hline Mujer & 5.478 & & $62 \%$ & 244 & & $48 \%$ \\
\hline \multicolumn{7}{|l|}{ Edad } \\
\hline Promedio & 52 & 11 & & 56 & 8 & \\
\hline \multicolumn{7}{|l|}{$\begin{array}{l}\text { Años de experiencia en } \\
\text { establecimientos }\end{array}$} \\
\hline Promedio & 24 & 13 & & 30 & 10 & \\
\hline \multicolumn{7}{|l|}{ Dependencia administrativa } \\
\hline Municipal & 4.609 & & $52 \%$ & 288 & & $52 \%$ \\
\hline Particular subvencionado & 4.255 & & $48 \%$ & 216 & & $48 \%$ \\
\hline \multicolumn{7}{|l|}{ Formación inicial } \\
\hline Educación párvulos & 984 & & $11 \%$ & 20 & & $4 \%$ \\
\hline Educación diferencial & 1.403 & & $16 \%$ & 31 & & $6 \%$ \\
\hline Educación general básica & 4.211 & & $48 \%$ & 284 & & $56 \%$ \\
\hline Educación media & 2.128 & & $24 \%$ & 183 & & $36 \%$ \\
\hline Área distinta a educación & & & $2 \%$ & 8 & & $2 \%$ \\
\hline Total & 8.864 & & 100 & 504 & & 100 \\
\hline
\end{tabular}

Fuente: Elaboración propia sobre la base de Idoneidad docente, 2015 (Mineduc, 2015) y la encuesta La voz de los directores, 2015. 
34 ¿CUÁN PREPARADOS LLEGAN LOS DIRECTORES ESCOLARES?: UN ANÁLISIS SOBRE SU FORMACIÓN Y TRAYECTORIAS LABORALES PREVIAS A EJERCER SU CARGO - R. Rivero, C. Hurtado, Á. Morandé

\section{Análisis}

Para abordar los objetivos de este estudio, se describen y analizan las distribuciones de formación, trayectorias laborales y el grado de preparación para ejercer un liderazgo efectivo, antes de llegar al cargo, considerando las dimensiones establecidas en el marco. Luego se caracteriza a los directores según el grado de preparación, el tipo de experiencia laboral y el tiempo en establecimientos antes de ser director. Para estudiar la preparación de los directores antes de ejercer el cargo en el tiempo, se compara la variable "Grado de preparación" entre los directores seleccionados por primera vez en los últimos 10 años con aquellos seleccionados hace más de 10 años. Por último, se estudia si hay diferencias en el grado de preparación entre directores municipales seleccionados vía ADP versus quienes que no han sido seleccionados por esta vía, comparando el grado de preparación de ambos grupos. Todos los grupos de comparación se caracterizan según los tipos de experiencia y los años de experiencia en establecimientos.

\section{Resultados}

\section{1. ¿Cuán preparados están los directores para ejercer el cargo por primera vez?}

En cuanto a la formación continua, la Tabla 2 muestra que el 90\% de los directores cursó algún programa de formación continua. Un $32 \%$ alcanzó cursos de perfeccionamiento, diplomado o postítulo como grado más alto y un 58\% terminó programas de magíster o doctorado (posgrado). Esta última proporción es similar a lo señalado en otros estudios nacionales (Weinstein y Muñoz, 2012). En cuanto al contenido u orientación de estos programas, el 22\% completó programas de posgrado relacionados con el liderazgo o gestión escolar, mientras que un 36\% en algún tema de educación (evaluación, currículo, entre otros). Considerando que los contenidos de los programas de posgrado en liderazgo y gestión apuntan a formar las competencias y habilidades para ejercer un liderazgo escolar efectivo, y la intensidad y duración de los programas de magíster y doctorado suele ser mayor, destaca que un alto porcentaje (78\%) de 
los directores no cursó un posgrado en el área, siendo predominante la formación continua en posgrados en otras áreas y/o en programas de formación de menor intensidad.

Tabla 2

Formación continua de los directores

\begin{tabular}{lcc}
\hline Formación continua & Número & Porcentaje \\
\hline Sin formación continua. & 48 & $10 \%$ \\
Diplomado, curso de perfeccionamiento o postítulo en & 164 & $32 \%$ \\
liderazgo o educación. & 109 & $22 \%$ \\
Magíster o doctorado en liderazgo/ gestión. & 183 & $36 \%$ \\
Magíster o doctorado en educación. & 504 & $100 \%$ \\
\hline Total
\end{tabular}

Fuente: Elaboración propia.

En relación con los cargos, la Tabla 3 muestra que un 9\% de los directores no se desempeñó previamente ni como profesor de aula ni en cargos de liderazgo intermedio en establecimientos. Por otra parte, un 26\% sí ejerció como profesor de aula, pero no en cargos de liderazgo intermedio. Estos resultados evidencian que el 35\% de los directores no tuvo experiencias laborales previas para formarse y desarrollar competencias y habilidades clave del liderazgo escolar. Por su parte, se destaca que el $65 \%$ de los directores tuvo ambas experiencias.

Tabla 3

Trayectoria laboral antes de ejercer el cargo como director

\begin{tabular}{lcc}
\hline Formación continua & Número & Porcentaje \\
\hline Sin formación continua. & 48 & $10 \%$ \\
Diplomado, curso de perfeccionamiento o postítulo en & 164 & $32 \%$ \\
liderazgo o educación. & 109 & $22 \%$ \\
Magíster o doctorado en liderazgo/gestión. & 183 & $36 \%$ \\
Magíster o doctorado en educación. & 504 & $100 \%$ \\
Total &
\end{tabular}

Nota: Se incluyen casos que tuvieron experiencia en cargos de liderazgo intermedio y no aula (2\% de la muestra).

Fuente: Elaboración propia. 
Para conocer cuán preparados llegan los directores a ejercer su cargo, la Tabla 4 combina la formación continua y las trayectorias laborales de los directores, describiendo los resultados de la variable "Nivel de preparación", que distingue tres categorías. Los resultados muestran que más de un cuarto de los directores presenta baja preparación para ejercer un liderazgo escolar efectivo, es decir, no tiene ni formación especializada en liderazgo escolar ni experiencia laboral en cargos de liderazgo intermedio, por lo que difícilmente puede contar con las competencias para ejercer un liderazgo efectivo previo a ejercer el cargo. Como se detalló anteriormente, la evidencia señala que son la formación especializada y, sobre todo, la experiencia profesional anterior, los espacios más relevantes para la formación de habilidades y competencias. De este grupo, un 39\% de los directores cursó un magíster en educación, pero no cuenta con formación especializada en liderazgo o gestión. En un segundo grupo, denominado de mediana preparación, se incluye a quienes cursaron posgrados especializados de liderazgo, pero no ejercieron cargos de liderazgo intermedio en escuelas, y también a quienes ejercieron cargos de liderazgo, pero no cuentan con formación de posgrado especializada. Este grupo es el mayor y alcanza un 60\% de los directores. Mientras que solo un 13\% de esta categoría debe su preparación a la consecución de posgrados especializados en liderazgo, el $87 \%$ debe esta clasificación a su experiencia laboral en cargos de liderazgo intermedio. De este grupo, solo un 14\% de los directores cursó un magíster en educación y no tiene experiencia laboral en cargos intermedios. Por último, el 13\% de los directores se encuentra altamente preparado, ya que declaran ambos espacios formativos, tanto formación especializada como experiencia laboral.

Tabla 4

Grado de preparación según formación continua y trayectoria laboral

\begin{tabular}{lcc}
\hline Grado de preparación & Número & Porcentaje \\
\hline Baja & 136 & $27 \%$ \\
Mediana & 302 & $60 \%$ \\
Alta & 66 & $13 \%$ \\
Total & 504 & $100 \%$ \\
\hline
\end{tabular}

Fuente: Elaboración propia 
Para profundizar en las experiencias de preparación previas al rol de director, la Tabla 5 caracteriza el grado de esta según los años de experiencia laboral, distinguiendo el tiempo dedicado al ámbito educacional, fuera de este y en establecimientos educacionales. Al comparar entre los grupos según el nivel de preparación, las mayores diferencias se encuentran en el tiempo dedicado a trabajar en establecimientos y en la edad en que comenzaron a ejercer el cargo. Esto es consistente con la construcción de los grupos de preparación. Por ejemplo, los directores de baja preparación tienen en promedio ocho años menos de experiencia en establecimientos y comenzaron tres años más jóvenes a ejercer el cargo.

Por su parte, los tres grupos de nivel de preparación coinciden en que dedicaron pocos años a empleos no relacionados con educación, o bien en educación, pero fuera de establecimientos educacionales (uno o dos años, en promedio). A su vez, destaca que menos del 30\% de los directores del grupo de baja preparación tuvo este tipo de experiencias laborales. Si bien desconocemos las características específicas en estos cortos periodos de tiempo, su baja prevalencia apunta a que no serían significativas para la formación de competencias directivas.

En relación con los años de experiencia en establecimientos educacionales, los resultados muestran una alta heterogeneidad. Los directores con baja preparación tienen en promedio 12 años previos a ejercer como directores versus 20 y 17 años, en los grupos de mediana y alta preparación, respectivamente. Específicamente, la Tabla 5 muestra que los directores de baja preparación se concentran en la categoría "fast", (51\%), los medianamente preparados presentan mayor dispersión, pero cierta concentración en la categoría "medium" (41\%) y, en segundo lugar, en "low" (37\%). Dicha dispersión es coherente con la varianza de la edad en que los directores ejercen por primera vez (la edad en que comienzan el cargo va desde los 34 a los 52 años). Por último, los directores del grupo de alta preparación presentan una alta dispersión, ya que un tercio alcanzó el puesto en menos de 10 años, un $40 \%$ lo hizo entre 11 y 24 años y un $26 \%$ se demoró 25 años o más. 
Estos resultados sugieren que el nivel de formación y experiencia en cargos escolares deseables para la formación de competencias directivas se relaciona con el periodo de tiempo que han destinado previamente al ámbito educacional, siendo menor la experiencia en establecimientos entre directores de baja preparación. Aun así, al observar la distribución del grupo de alta preparación, se evidencia que este se compone de casos con diversos años de trayectorias previas.

Tabla 5

Grado de preparación y tipos de experiencia laboral

\begin{tabular}{llrrrr}
\hline & & Baja & Mediana & Alta & Total \\
\hline Años experiencia laboral no & Promedio & 2 & 1 & 1 & 1 \\
educación. & Desviación estándar & 4 & 3 & 2 & 3 \\
Años experiencia en educación, & Promedio & 1 & 1 & 0 & 1 \\
pero no en establecimientos. & Desviación estándar & 3 & 2 & 1 & 2 \\
Años experiencia en & Promedio & 12 & 20 & 17 & 17 \\
establecimientos. & Desviación estándar & 10 & 10 & 9 & 10 \\
Edad en que se hizo director. & Promedio & 40 & 45 & 42 & 43 \\
& Desviación estándar & 9 & 9 & 8 & 9 \\
Experiencia fuera del ámbito & Número & 25 & 38 & 16 & 79 \\
educacional. & Porcentaje & 18 & 14 & 16 & 16 \\
Experiencia en el ámbito & Número & 18 & 29 & 4 & 51 \\
educacional, pero no en & Porcentaje & 13 & 11 & 4 & 10 \\
establecimientos. & Número & 70 & 59 & 30 & 159 \\
"Fast" (10 o menos años). & Porcentaje & 51 & 22 & 30 & 32 \\
& Número & 49 & 110 & 43 & 202 \\
& Porcentaje & 36 & 41 & 43 & 40 \\
"Medium" (entre 11 y 24 años) & Número & 17 & 100 & 26 & 143 \\
"Low" (25 años o más). & Porcentaje & 13 & 37 & 26 & 28 \\
\hline
\end{tabular}

Fuente: Elaboración propia. 
4.2. ¿Ha cambiado el perfil de director en relación con su preparación para ejercer el cargo en la última década y según el proceso de selección de los directores?

Para comprender los posibles cambios en el grado de preparación para el cargo de director — definido por su experiencia laboral y formación especializada - la Tabla 6 presenta esta variable según el año de nombramiento como director en el establecimiento actual, aquellos nombrados antes del año 2005, es decir, hace más de 10 años, en comparación con aquellos nombrados desde el año 2005 en adelante, es decir, hace 10 años o menos. A su vez, se presenta la comparación del grado de preparación entre directores municipales seleccionados por ADP y aquellos seleccionados por otro método.

Los resultados muestran que el grado de preparación (que combina la formación y trayectoria laboral en establecimientos) ha mejorado en la última década, disminuyendo un 16\% los directores con baja preparación y aumentado un $8 \%$ los medianamente preparados y un $8 \%$ los altamente preparados. Por el contrario, los directores de establecimientos municipales seleccionados por ADP son muy similares en su grado de preparación a aquellos no seleccionados bajo esta política ADP.

Tabla 6

Grado de preparación según año de nombramiento y sistema de selección

\begin{tabular}{lrrrrrrrr}
\hline & \multicolumn{3}{c}{ Año nombramiento director } & \multicolumn{3}{c}{ Sistema de selección director } \\
\hline Grado de preparación & \multicolumn{1}{c}{ Antes } & 2005 & \multicolumn{2}{c}{2005} & -2015 & No ADP & \multicolumn{2}{c}{ ADP } \\
\hline \multirow{2}{*}{ Baja } & $\mathrm{N}$ & $\%$ & $\mathrm{~N}$ & $\%$ & $\mathrm{~N}$ & $\%$ & $\mathrm{~N}$ & $\%$ \\
Mediana & 83 & 35 & 53 & 19 & 52 & 29 & 32 & 30 \\
Alta & 115 & 49 & 154 & 57 & 95 & 53 & 59 & 55 \\
Total & 36 & 15 & 63 & 23 & 33 & 18 & 17 & 16 \\
\hline
\end{tabular}

Fuente: Elaboración propia.

Por su parte, la Tabla 7 caracteriza los años de experiencia laboral en educación, fuera de la educación y en establecimientos educacionales, para los cuatro grupos de directores. Los resultados muestran que la experiencia laboral fuera del ámbito de la educación 
y fuera de establecimientos educacionales es similar entre directores que comenzaron a ejercer en distintas décadas. Sin embargo, el porcentaje de directores seleccionados con experiencia fuera del ámbito de la educación disminuye de un 24\% a un 6\%, al comparar ambos periodos de nombramientos. En relación con los años promedio en establecimientos educacionales, los resultados muestran que los directores nombrados hace más de diez años tienen una experiencia promedio de 14 años, seis años menor que aquellos seleccionados en la última década. Consistentemente, los directores nombrados antes, alcanzaron en promedio el puesto a los 39 años en comparación con los 46 años de edad promedio del grupo nombrado en los últimos diez años. Asimismo, los directores nombrados antes de 2005, se concentran en la trayectoria "fast", mientras que los elegidos posteriormente lo hacen en la categoría "low"; en ambos casos, alrededor de un $40 \%$ corresponde a la categoría de quienes fueron nombrados tras trabajar entre 11 y 24 años en establecimientos ("medium").

Por último, el análisis de la experiencia laboral de los directores municipales según el proceso de selección, muestra similitud entre los grupos con relación a su trayectoria laboral. La principal diferencia se observa en que el 19\% de los directores seleccionados por ADP cuenta con años de experiencia fuera de la educación en comparación con el $9 \%$ de aquellos no seleccionados por este procedimiento. Adicionalmente, se observan algunas diferencias en los años de experiencia en establecimientos, siendo levemente mayor la proporción de directores seleccionados por ADP pertenecientes a la categoría "fast" al comparar con quienes no fueron seleccionados por este método. 
Tabla 7

Tipos de experiencia laboral según año de nombramiento y sistema de selección

\begin{tabular}{|c|c|c|c|c|c|}
\hline & & \multicolumn{2}{|c|}{$\begin{array}{c}\text { Año nombramiento } \\
\text { director }\end{array}$} & \multicolumn{2}{|c|}{$\begin{array}{c}\text { Sistema de selecciór } \\
\text { director }\end{array}$} \\
\hline & & $\begin{array}{l}\text { Antes } \\
2005\end{array}$ & $\begin{array}{l}2005- \\
2015\end{array}$ & No ADP & ADP \\
\hline \multirow{2}{*}{$\begin{array}{l}\text { Años experiencia laboral no } \\
\text { educación. }\end{array}$} & Promedio & 1 & 1 & 0 & 1 \\
\hline & $\mathrm{DE}$ & 3 & 3 & 1 & 4 \\
\hline \multirow{2}{*}{$\begin{array}{l}\text { Años experiencia en } \\
\text { educación. }\end{array}$} & Promedio & 1 & 1 & 0 & 1 \\
\hline & $\mathrm{DE}$ & 2 & 2 & 2 & 2 \\
\hline \multirow{2}{*}{$\begin{array}{l}\text { Años experiencia } \\
\text { en establecimientos } \\
\text { educacionales. }\end{array}$} & Promedio & 14 & 20 & 20 & 18 \\
\hline & $\mathrm{DE}$ & 9 & 10 & 10 & 10 \\
\hline \multirow{2}{*}{$\begin{array}{l}\text { Edad en que } \\
\text { se hizo director. }\end{array}$} & Promedio & 39 & 46 & 44 & 45 \\
\hline & $\mathrm{DE}$ & 8 & 8 & 9 & 9 \\
\hline \multirow{2}{*}{$\begin{array}{l}\text { Fuera del ámbito } \\
\text { educacional. }\end{array}$} & Número & 64 & 15 & 20 & 21 \\
\hline & Porcentaje & $24 \%$ & $6 \%$ & $11 \%$ & $19 \%$ \\
\hline \multirow{2}{*}{$\begin{array}{l}\text { En el ámbito educacional, } \\
\text { pero no en establecimientos. }\end{array}$} & Número & 20 & 31 & 13 & 19 \\
\hline & Porcentaje & $9 \%$ & $11 \%$ & $7 \%$ & $18 \%$ \\
\hline \multirow{2}{*}{ "Fast" (10 o menos años). } & Número & 103 & 56 & 41 & 33 \\
\hline & Porcentaje & $44 \%$ & $20 \%$ & $23 \%$ & $31 \%$ \\
\hline \multirow{2}{*}{$\begin{array}{l}\text { "Medium" (entre } 11 \text { y } 24 \\
\text { años). }\end{array}$} & Número & 95 & 107 & 70 & 41 \\
\hline & Porcentaje & $41 \%$ & $40 \%$ & $39 \%$ & $38 \%$ \\
\hline \multirow{2}{*}{ "Low" (25 años o más). } & Número & 36 & 107 & 69 & 34 \\
\hline & Porcentaje & $15 \%$ & $40 \%$ & $38 \%$ & $31 \%$ \\
\hline \multirow[t]{2}{*}{ Total } & Número & 234 & 270 & 180 & 108 \\
\hline & Porcentaje & $100 \%$ & $100 \%$ & $100 \%$ & $100 \%$ \\
\hline
\end{tabular}

Fuente: Elaboración propia.

\section{Conclusiones}

Los esfuerzos de investigación que evalúan la preparación de los directores para ejercer un liderazgo efectivo son recientes y valiosos para conocer brechas y espacios de mejora, asegurando el ejercicio de un liderazgo efectivo. Estudios anteriores sugieren que los directores 
presentan debilidades en aquellas áreas más importantes según la literatura, como es el foco en las funciones pedagógicas (Marfán et al., 2012; Volante, 2012) y de gestión (CIDE, 2014), entre ellas, la formación para dirigir procesos de enseñanza y construir una visión estratégica y conocimientos sobre bases curriculares y estándares de aprendizaje y, sobre liderazgo escolar (CEDLE, 2017; Weinstein et al., 2016). Este estudio analiza la formación continua y las experiencias laborales previas de los directores al ejercer el cargo por primera vez, considerando ambos espacios como los principales momentos de formación y desarrollo de habilidades y competencias requeridas para ejercer un liderazgo efectivo.

A partir de la opinión de los directores de establecimientos subvencionados en Chile, los resultados de este estudio revelan que un grupo importante de ellos entran al cargo por primera vez con una baja preparación para ejercer un liderazgo efectivo. Esta conclusión se fundamenta en que uno de cada cuatro directores no cuenta con ninguna experiencia formativa clave antes de ejercer por primera vez, es decir, no ha cursado un posgrado especializado en liderazgo o gestión escolar, ni tiene experiencia laboral en cargos de liderazgo escolar intermedio, siendo coherente con otros resultados de la literatura respecto de las debilidades de los directores, en especial en las áreas pedagógicas y de gestión. Adicionalmente, destaca de forma negativa que más de un tercio de los directores encuestados no posee experiencia en cargos de liderazgo escolar y solo un $13 \%$ se encuentra altamente preparado, ya que cuenta con ambas experiencias de preparación (posgrado y experiencia laboral). Por otra parte, las trayectorias laborales previas, fuera de establecimientos educacionales, son escasas, y presentes en un grupo bajo de directores, por pocos años (uno o dos), evidenciando que son espacios poco relevantes en su formación y desarrollo profesional para ejercer un liderazgo efectivo.

Al analizar la tendencia histórica de la preparación para ejercer el cargo de director por primera vez, los resultados muestran que esta ha mejorado en la última década en comparación con la situación previa al año 2005, disminuyendo un 16\% los directores con baja preparación y aumentando en seis años la experiencia laboral en 
establecimientos y los años totales de experiencia laboral antes de ser director. Si bien el grado de preparación ha mejorado en la última década, ya que cuentan con un mayor grado de formación y experiencia laboral, los resultados sugieren que todavía un porcentaje importante de directores de establecimientos subvencionados comienza a ejercer el cargo con una baja preparación. Por otra parte, al analizar las diferencias en el grado de preparación de los directores municipales, no se observaron diferencias entre aquellos directores seleccionados por ADP y aquellos que no, difiriendo de resultados encontrados por otros estudios que concluyen que los directores seleccionados por ADP tienen menor experiencia laboral y mayor formación especializada que aquellos no seleccionados. En otras palabras, los datos sugieren que la mejora en el grado de preparación en la última década no se asocia a los cambios generados en las trayectorias laborales de los directores seleccionados por ADP. Estos resultados son coherentes con los requisitos mínimos establecimientos por ADP para ser director.

Si bien los resultados y conclusiones hay que interpretarlos con cautela, estos permiten concluir que existe un amplio espacio de mejora tanto para los programas de formación especializada como para la política educativa de liderazgo escolar. En primer lugar, la baja prevalencia de formación de posgrado especializada en los directores, sumado a la evidencia de que esta formación, cuando tiene un componente fuerte centrado en la formación práctica se asocia positivamente a ejercer un liderazgo efectivo, apuntan a la necesidad de aumentar la formación especializada para directores. Para que este aumento en la formación tenga un real impacto en el liderazgo y el aprendizaje de los estudiantes, es importante que sean espacios formativos que enfaticen con un enfoque desde la práctica, la dimensión pedagógica y los conocimientos, habilidades y prácticas asociadas al MBDLE, como también que considere los contextos educativos en los cuales los directores se insertarán. A su vez, es importante avanzar hacia la evaluación de estos programas, ya que en el contexto chileno se cuenta con escasa evidencia de su efectividad y, por lo tanto, aumentar la formación de posgrado especializada sin poseer información respecto de su calidad podría no generar los efectos deseados en el liderazgo y el aprendizaje de los estudiantes. 
En segundo lugar, los resultados de este estudio son un potencial insumo para el desarrollo de otras políticas de liderazgo escolar, como también para mejorar la coherencia entre las actuales políticas educativas. Por una parte, la baja preparación de los directores evidenciada - más de un tercio no tuvo ninguna experiencia en liderazgo antes de ejercer por primera vez como director- sugiere la creación de una carrera directiva como una alternativa para mejorar la preparación de los directores para asumir sus funciones por primera vez. Por ejemplo, una carrera directiva podría incluir como requisito mínimo que los directores que ejerzan por primera vez en establecimientos subvencionados tengan obligatoriamente un número de años de experiencia laboral previa en cargos directivos. En este sentido, la evidencia internacional es concluyente en señalar que la experiencia en cargos directivos anteriores es clave para el desarrollo profesional de las competencias y habilidades de liderazgo y, a su vez, los directores chilenos confirman esta evidencia. Si bien dicha experiencia laboral en liderazgo intermedio no es el único camino para la preparación hacia un liderazgo efectivo, el diseño de una carrera directiva y, en particular, la dimensión sobre selección de directores novatos, podría considerar la experiencia previa como un factor clave dentro del desarrollo profesional de los futuros directores. Por otra parte, como política complementaria para los directores novatos sin experiencia laboral en cargos directivos, podría diseñarse un plan de apoyo de mentorías de manera de suplir, en parte, la preparación con la que directores llegan a ejercer el cargo por primera vez.

Los resultados de este estudio son un importante insumo para la implementación de una carrera directiva. Por ejemplo, si bien los resultados sugieren avanzar en esa línea, difícilmente se puede crear una carrera que en el corto plazo tenga como requisito mínimo contar con un número de años de experiencia en cargos de liderazgo intermedio o poseer una certificación de competencias o programa de formación especializada antes de ejercer, ya que sería muy difícil de cumplir para los actuales directores de establecimientos subvencionados. Los datos de este estudio muestran que más de un $35 \%$ de los directores no cumplirían con el requisito de experiencia laboral previa en cargos directivos y un $78 \%$ no cumplirían con el requisito de una formación especializada. 
A su vez, es importante destacar que la revisión de literatura, los resultados y conclusiones de este estudio no son consistentes con el diseño actual de algunas políticas educacionales vigentes, las que incentivan la experiencia laboral previa como docente de aula por sobre la formación y otras trayectorias laborales. En particular, la Ley de Calidad y Equidad de la Educación establece un mínimo de tres años de experiencia en aula para directores de establecimientos municipales. Por otra parte, la reciente Ley de Carrera Docente especifica que, a partir de este año, solo los profesores que se encuentren en el tramo avanzado, es decir, que tengan una evaluación sobresaliente en habilidades, conocimientos y prácticas definidas en el MBDLE, pueden acceder a ser director en establecimientos municipales. Sin embargo, las competencias de un profesor efectivo no son equivalentes a las identificadas por el MBDLE para ejercer un liderazgo efectivo. En otras palabras, si bien la Ley de Carrera Docente es un avance para el desarrollo profesional docente, resulta una política no articulada con el aseguramiento de la calidad de la preparación de los directivos. El desarrollo profesional directivo debería estar vinculado a su formación especializada y al desarrollo profesional en cargos de liderazgo intermedio, ya sea como jefe de ciclo, coordinador, jefe de UTP u otros en los cuales se puedan desarrollar las competencias requeridas para ejercer como director de forma efectiva según el MBDLE.

Finalmente, sería interesante expandir esta línea de investigación. Este estudio concluye que existe una amplia diversidad de formación y trayectorias entre quienes lideran las escuelas del país. Futuras investigaciones deben ahondar en la relación entre la preparación, es decir, la formación especializada y experiencia y el liderazgo escolar efectivo y el aprendizaje de los estudiantes. En esta línea, se debe progresar en investigaciones acerca de cómo las experiencias previas de formación y las trayectorias preparan a los directores en las habilidades y conocimientos señalados en el MBLDE y en prácticas efectivas. Si bien, el conocimiento de programas de formación especializada es extenso, se desconoce cómo ambas dimensiones (formación continua y trayectorias) aportan a la preparación. Por otra parte, será relevante estudiar el grado de preparación de los directores que ejercen el cargo por primera vez en 
el tiempo, estudiando cuán preparados están y cuál es la tendencia en este aspecto. Por ejemplo, en la actualidad, la Ley de Carrera Docente plantea como más atractivo para algunos profesores trabajar como profesor de aula que como director o en cargos directivos, y a su vez, transforma el perfil de candidato a director de establecimiento municipal. Estos cambios normativos pueden tener consecuencias en el perfil de los candidatos que postulan y, por lo tanto, en la preparación efectiva, formación especializada y trayectoria laboral previa para ejercer. Por último, otros estudios podrán profundizar en las motivaciones de docentes y/o en los contextos en que deciden transitar a cargos directivos para comprender la el paso a la carrera directiva. Un estudio de Wallace Foundation (Saltzman, 2016) encontró que el $80 \%$ de los asistentes de dirección en EE.UU. aspira a ejercer como directores. También se desconocen las trayectorias tras ser nombrados para el cargo, particularmente, patrones de rotación o abandono temprano, como sucede en otros sistemas.

Con todo, este estudio y otros, permitirán generar mayor evidencia e identificar desafíos para desarrollar una carrera directiva, políticas de formación y selección de directores, y otras políticas de liderazgo que consideren la formación especializada, la trayectoria laboral de futuros directores y la definición de prácticas necesarias (como sería la inclusión de mentorías en la formación o la obligatoriedad de la inducción), para asegurar que los directivos desplieguen todas las habilidades de liderazgo necesarias. Ante mayores demandas y expectativas, urge generar más evidencia acerca de la preparación para conformar los equipos que lideran las escuelas, como también ampliar estos estudios a otros miembros de los equipos directivos.

\section{Referencias}

Agencia de Calidad de la Educación (2016). Directores municipales seleccionados mediante la Ley $N^{\circ} 20.501$. Santiago de Chile: Autor.

Barber, M. \& Mourshed, M. (2007). How the world's best-performing schools come out on top. London: McKinsey and Company.

Bowers, J. \& Bradford, R. (2014). Do principal preparation and teacher qualifications influence different types of school growth trajectories 
in Illinois? A growth mixture model analysis. Journal of Educational Administration, 52(5), 705-736.

https://doi.org/10.1108/JEA-12-2012-0134

Centro de Desarrollo de Liderazgo Educativo, CEDLE. (2016). Resultados encuesta "La voz de los directores". Santiago de Chile: Universidad Diego Portales.

Clark, D., Martorell, P., \& Rockoff, J. (2009). School principals and school performance. (Working Paper 38). Washington, DC.: National Center for Analysis of Longitudinal Data in Education Research.

Errázuriz, M., Kutscher, M., y Williamson, C. (2016). La Ley 20.501 sobre Calidad y Equidad en los colegios públicos: efectos de la selección de directores por la Alta Dirección Pública (ADP) (Documento de trabajo). Santiago de Chile: CLAPES UC

Farley-Ripple, E., Mead, H., Raffel, J., Sherretz, K., \& Welch, J. (2011). Tracking transitions. Nueva York: Delaware Academy for School Leadership. Wallace Foundation.

Gates, S. M., Ringel, J. S., \& Santibanez, L. (2003). Who is leading our schools?: An overview of school administrators and their careers. Recuperado de https://www.rand.org/pubs/monograph_reports/MR1679.html.

Grupo Educativo (2015). Evaluación de la implementación del sistema de selección de directores en el marco de la Ley $N^{\circ}$ 20501. Santiago de Chile: Autor.

Hale, E. L. \& Moorman, H. N. (2003). Preparing school principals: A national perspective on policy and program innovations. Washington, DC.: Institute for Educational Leadership (NJ1).

Hallinger, P. \& Heck, R. H. (1998). Exploring the principal's contribution to school effectiveness: 1980-1995. School effectiveness and school improvement, 9(2), 157-191.

http://dx.doi.org/10.1080/0924345980090203

Harris, A. (2009). Distributed leadership: What we know. Recuperado de https://www.researchgate.net/publication/226009218_Distributed_ Leadership_What_We_Know

Leithwood, K., Day, C., Sammons, P., Harris, A., \& Hopkins, D. (2006). Successful school leadership: What it is and how it influences pupil learning. London: DfES and Nottingham: NCSL.

Ley $N^{\circ} 19.070$ Aprueba Estatuto de los Profesionales de la Educación del Ministerio de Educación. Diario Oficial de la República de Chile, 1 de julio de 1991. 
48 ¿CUÁN PREPARADOS LLEGAN LOS DIRECTORES ESCOLARES?: UN ANÁLISIS SOBRE SU FORMACIÓN Y TRAYECTORIAS LABORALES PREVIAS A EJERCER SU CARGO - R. Rivero, C. Hurtado, Á. Morandé

Ley N 20.370 Establece la Ley General de Educación del Ministerio de Educación. Diario Oficial de la República de Chile, 12 de septiembre de 2009.

Ley No 20.903 Crea el Sistema de Desarrollo Profesional Docente y Modifica otras normas. Diario Oficial de la República de Chile, 1 de abril de 2016.

Ley N²0.501 Calidad y Equidad de la Educación del Ministerio de Educación. Diario Oficial de la República de Chile, 26 de febrero 2011.

Loeb, S., Kalogrides, D., \& Horng, E. L. (2010). Principal preferences and the uneven distribution of principals across schools. Educational Evaluation and Policy Analysis, 32(2), 205-229. https://doi.org/10.3102/0162373710369833

Marfán, J., Pascual, J., Muñoz, G., González, R., Valenzuela, P. \& Weinstein, J. (2012). Estudio comparado de liderazgo escolar: Aprendizajes para Chile a partir de los resultados PISA 2009. Santiago de Chile: FONIDE, Ministerio de Educación.

Marzano, R. J., Waters, T., \& McNulty, B. (2005). School leadership that works: From research to results. Aurora, CO: ASCD and McREL.

Militello, M., Gajda, R., \& Bowers, A. J. (2009). The role of accountability policies and alternative certification on principals' perceptions of leadership preparation. Journal of Research on Leadership Education, 4(3), 30-66. https://doi.org/10.1177/194277510900400301

Ministerio de Educación de Chile, Mineduc. (2015). Base de datos de Idoneidad docente. Recuperado de http://datosabiertos.mineduc.cl

Ministerio de Educación de Chile (2015). Bases de Datos Directorio de Establecimientos Educacionales. Recuperado de: http://datosabiertos. mineduc.cl

Ministerio de Educación de Chile, Mineduc. (2015). Marco para la buena dirección, criterios para el desarrollo profesional y evaluación de desempeño. Santiago de Chile: Autor.

Núñez, I., Weinstein, J., y Muñoz, G. (2010). ¿Posición olvidada? Una mirada desde la normativa a la historia de la dirección escolar en chile. Psicoperspectivas, 9(2), 53-81.

http://dx.doi.org/10.5027/psicoperspectivas-Vol9-Issue2-fulltext-117

Pont, B., Nusche, D., \& Moorman, H. (2009). Improving school leadership. Volume 1: Policy and practice. Paris: OECD publishing.

Rice, J. K. (2010). Principal effectiveness and leadership in an era of accountability: What research says (Brief 8). Washington, DC.: National Center for Analysis of Longitudinal Data in Education Research. 
Robinson, V. M., Lloyd, C. A., \& Rowe, K. J. (2008). The impact of leadership on student outcomes: An analysis of the differential effects of leadership types. Educational administration quarterly, 44(5), 635674. http://dx.doi.org/10.1177/0013161X08321509

Saltzman, A. (2016). The power of principals supervisors. Wallace Fundation. Recuperado de http://www.wallacefoundation.org/knowledge-center/ Documents/The-Power-of-Principal-Supervisors.pdf

Silins, H. \& Mulford, B. (2002). Schools as learning organizations: The case for system, teacher and student learning. Journal of educational administration, 40(5), 425-446.

http://dx.doi.org/10.1108/09578230210440285

Stevenson, H. (2006). Moving towards, into and through principal ship: Developing a framework for researching the career trajectories of school leaders. Journal of Educational Administration, 44(4), 408-420. https://doi.org/10.1108/09578230610676604

Volante, P. (2012). Liderazgo instruccional y logro académico en la educación secundaria en Chile. En J. Weinstein y G. Muñoz (Eds.), ¿Qué sabemos sobre los directores de escuela en Chile? (pp. 349-370). Santiago de Chile: Centro de Estudios de Políticas y Prácticas en Educación.

Weinstein, J., Cuéllar, C., Hernández, M., y Fernández, M. (2016). Director(a) por primera vez: un estudio sobre la experiencia y socialización de los directores noveles en establecimientos municipales de Chile. Calidad en la Educación, 44, 12-45.

http://dx.doi.org/10.4067/S0718-45652016000100002

Weinstein, J. y Hernández, M (2014). Políticas hacia el liderazgo directivo escolar en Chile: una mirada comparada con otros sistemas escolares de América Latina. Psicoperspectivas, 13(3), 52-68.

Weinstein, J. y Muñoz, G. (2012). Cuando las atribuciones no bastan: liderazgo directivo y gestión pública o privada de escuelas en chile. En J. Weinstein y G. Muñoz (Eds.), ¿Qué sabemos sobre los directores de escuela en Chile? (pp. 55-83). Santiago de Chile: Centro de Estudios de Políticas y Prácticas en Educación.

Weinstein, J., Muñoz, G., y Hernández, M. (2014). El liderazgo escolar en América Latina y el Caribe: un estado del arte en base a ocho sistemas escolares de la región. Santiago de Chile: OREALC/Unesco.

Recibido: 27/11/2017

Aceptado: 04/06/2018 